\title{
Obstructive Sleep Apnea Syndrome, Hypoxemia and Endothelial Dysfunction: One Disease or Many? \\ Chitra Lal $^{1 *}$, Charlie Strange ${ }^{2}$
}

${ }^{1}$ Assistant Professor of Medicine, Medical University of South Carolina, Division of Pulmonary, Critical Care, Allergy and Sleep Medicine, 96 Jonathan Lucas Street, CSB 812, MSC 630, Charleston, SC 29425, USA

${ }^{2}$ Professor of Medicine, Medical University of South Carolina, Division of Pulmonary, Critical Care, Allergy and Sleep Medicine, 96 Jonathan Lucas Street, CSB 812, MSC 630, Charleston, SC 29425, USA

\begin{abstract}
The significant adverse sequelae of Obstructive Sleep Apnea Syndrome (OSAS) include a higher incidence of stroke, myocardial infarction and neurocognitive deficits. A link between the intermittent hypoxia of OSAS and endothelial dysfunction may explain many of the macrovascular and microvascular complications of OSAS. The pathogenesis of endothelial dysfunction involves an alteration in the levels of pro-inflammatory and pro-atherogenic mediators. As a form of ischemia/reperfusion injury to the endothelium, intermittent hypoxia induces reactive oxygen species and dysregulates vasoactive metabolites including pathways of nitric oxide synthesis. In addition, hypercoagulability and altered leucocyte migration contribute to the spectrum of endothelial dysfunction. The tools for measurement of endothelial dysfunction and its clinical implications are discussed. Endothelial dysfunction can be in part reversed with continuous positive airway pressure. Thus, early recognition and aggressive treatment of OSAS may prevent associated endothelial dysfunction and subsequent complications of this syndrome.
\end{abstract}

Keywords: Hypoxemia; Endothelial dysfunction; Obstructive sleep apnea syndrome; Atherosclerosis

\begin{abstract}
Abbreviations: OSAS: Obstructive Sleep Apnea Syndrome; VEGF Vascular Endothelial Growth Factor; IL-8: Interleukin 8; TNF-alpha: Tumor Necrosis Factor-alpha; NO: Nitric Oxide; ICAM-1: Intercellular Adhesion Molecule-1; VCAM-1: Vascular Cell Adhesion Molecule-1; EMPs: Endothelial Microparticles; EPC: Endothelial Progenitor Cell; CPAP: Continuous Positive Airway Pressure
\end{abstract}

\section{Introduction}

Obstructive Sleep Apnea Syndrome (OSAS) is a common disorder which is often underdiagnosed. Since OSAS is a major public health problem, significant interest has been generated in the health impact and long term sequelae of this disease. Common manifestations of OSAS include snoring, excessive daytime sleepiness and fatigue. In addition, emerging data has linked OSAS to neurocognitive deficits such as memory loss and attention deficits [1], hypertension, arrhythmias, coronary artery disease [2], congestive heart failure, stroke [3] and insulin resistance [4]. One link between the diverse clinical manifestations would be a shared pathogenesis associated with macrovascular or microvascular disease.

\section{Hypoxemia and endothelial dysfunction in OSAS}

OSAS causes recurrent episodic oxygen desaturation during sleep. Several studies suggest that OSAS also causes endothelial dysfunction $[5,6]$.

The endothelium is a metabolically active layer of cells lining the intima of blood vessels. Dysfunction of the endothelium can be defined as an alteration of any endothelial function including the transport of substances across the vessel wall, maintenance of vascular smooth muscle tone, secretion of anticoagulant factors, and expression of cell adhesion molecules which can cause granulocyte adhesion and migration.

Since endothelial cells line blood vessels, they are the first cells to be exposed to the effects of hypoxemia. The response of endothelium to stressors like hypoxia can be adaptive or maladaptive. An adaptive response may prolong cellular survival. A maladaptive response on the other hand, may perpetuate cellular injury and cause adverse sequelae like stroke, myocardial infarction and death [7]. Examples of a maladaptive response include the development of increased vascular permeability after exposure to hypoxia, presumably due to stimulation of vascular endothelial growth factor (VEGF). Hypoxia can also cause a hypercoagulable state, perhaps through the suppression of thrombomodulin which promotes the production of activated protein C.

In addition, intermittent hypoxemia increases levels of a transcription factor, nuclear factor kappa B [8] which upregulates the production of pro-inflammatory and pro-atherogenic mediators like interleukin 8 (IL-8) [9], and tumor necrosis factor-alpha (TNF-alpha) [8]. These in turn, may result in an accelerated atherosclerotic response.

The endothelium also produces several vasoactive mediators such as nitric oxide (NO), prostacyclin, endothelin-1 and thromboxane. While $\mathrm{NO}$ and prostacyclin have vasodilator properties, endothelin-1 and thromboxane are vasoconstrictors. An imbalance of these factors can alter the vascular smooth muscle tone [10]. OSAS decreases endothelial nitric oxide synthase activity [11]. Thus, a heightened vasoconstrictor response may decrease blood flow to critical organs such as the heart and the brain. In addition, NO has anti-inflammatory properties and inhibition of NO production can heighten the atherosclerotic response.

A high level of factors such as L-selectin, intercellular adhesion molecule-1(ICAM-1) and vascular cell adhesion molecule-1 (VCAM1) has been seen in OSAS patients [12]. These factors indicate an activated endothelium and may also play a role in the hormonal

*Corresponding author: Chitra Lal, MD, Assistant Professor of Medicine, Medica University of South Carolina, Division of Pulmonary, Critical Care, Allergy and Sleep Medicine, 96 Jonathan Lucas Street, CSB 812, MSC 630, Charleston, SC 29425, USA, Tel: 843792 7776; E-mail: lalch@musc.edu, chitra lal@hotmail.com

Received September 08, 2011; Accepted October 17, 2011; Published October 19, 2011

Citation: Lal C, Strange C (2011) Obstructive Sleep Apnea Syndrome, Hypoxemia and Endothelial Dysfunction: One Disease or Many? J Pulmonar Respirat Med 1:101. doi:10.4172/2161-105X.1000101

Copyright: (c) 2011 Lal C, et al. This is an open-access article distributed under the terms of the Creative Commons Attribution License, which permits unrestricted use, distribution, and reproduction in any medium, provided the original author and source are credited. 
imbalances, hypercoagulability and altered leukocyte migration which form a part of the spectrum of endothelial dysfunction.

Intermittent hypoxia followed by reoxygenation is a form of ischemia-reperfusion injury which has been implicated in local and remote tissue destruction. The mechanisms which have been implicated are an increased production of reactive oxygen species, increased markers of systemic inflammation and an altered balance of metabolites implicated in vascular tone.

Whether OSAS can trigger endothelial dysfunction independent of hypoxemia is unclear. Milder degrees of OSAS without significant nocturnal hypoxemia still are associated with adverse clinical sequelae including acute coronary syndrome and stroke. Since no large studies evaluating this specific question are available, this is an area requiring further study.

\section{Measurement of endothelial dysfunction}

Endothelial dysfunction has been measured in the OSAS population by alterations in microvascular flow or an increase in markers of endothelial damage or repair. Microvascular flow abnormalities have been seen using flow-mediated dilation of the brachial artery [13], pulse wave analysis, pulse wave velocity [14], and laser doppler flowmetry. Flow-mediated dilation of the vasculature is in part due to nitric oxide and occurs in response to an acute increase in blood flow.

Endothelial microparticles (EMPs) are small vesicles which are released from the endothelium in response to various stressors like intermittent hypoxia which can cause endothelial damage. They can be quantified by flow cytometry using specific antibodies against endothelial antigens. In a prospective study, EMPs were found to be elevated in OSAS patients as compared to controls [15]. Furthermore, the levels of EMPs correlated with the severity of OSAS.

Endothelial repair is in part mediated by a circulating endothelial cell called the endothelial progenitor cell (EPC) [16] that can be counted in blood. EPC numbers have been found to be variable in OSAS [17], which may be a reflection of additional factors influencing individual susceptibility to ischemia-reperfusion injury. Currently these tests of endothelial dysfunction are not commonly available and have been used mainly in research protocols.

\section{Clinical implications of endothelial dysfunction}

The link between endothelial dysfunction, microvascular pathology and macrovascular disease is critical to understand whether markers of endothelial dysfunction are predictive of clinical events. The hope would be that endothelial dysfunction may have great prognostic value as an early surrogate marker of atherosclerosis.

The prevalence of stroke and myocardial infarction in the OSAS population is quite high. In one case control study, the relative risk of stroke in snorers compared to non-snorers was 10.3 [18]. After adjustment for other risk factors like alcohol consumption, hypertension and coronary artery disease, the odds ratio for stroke in OSAS patients was found to be 8 [19]. A higher incidence of stroke in patients with sleep disordered breathing has been found in other studies as well $[20,21]$. OSAS has been found to be a strong risk factor for coronary artery disease also [22]. Thus the adverse impact of OSAS on health is considerable.

Endothelial dysfunction also can be seen in snorers and patients with mild OSAS [23] although a strong correlation has been found between the degree of endothelial dysfunction and the severity of OSAS $[24,25]$. Several studies have shown that endothelial dysfunction can be improved with the use of continuous positive airway pressure (CPAP) $[26,27]$. It would follow that some adverse consequences of OSAS such as stroke and myocardial infarction might be improved by CPAP therapy. A recent randomized, controlled trial assessing the impact of CPAP on ischemic stroke patients showed improved neurological and cardiovascular outcomes over a 2 year period of follow-up in those with moderate to severe OSAS who were initiated on CPAP early as compared to patients who were not treated with CPAP [28].

The role of endothelial dysfunction in pediatric patients with OSAS has also been studied. Endothelial dysfunction has been shown to be present in non-obese children with OSAS when compared to healthy controls [29]. Similar findings have been replicated in several other pediatric studies. On the other hand, a recent negative study showed endothelial dysfunction to be a function of obesity in prepubertal children rather than OSAS [30]. One explanation for this finding could be differences in individual susceptibility to the effects of the intermittent hypoxia seen in OSAS due to variable endothelial functional phenotypes and endothelial repair mechanisms [16].

With the currently available data, there is still much work to be done to further understand the mechanisms of endothelial dysfunction in OSAS. Important work remains to disentangle the many confounding comorbidities in the OSAS population that can independently cause abnormalities of endothelial function.

\section{Conclusion}

Intermittent hypoxemia seen in untreated OSAS is in part responsible for endothelial dysfunction. A strong link between endothelial dysfunction, microvascular flow alterations, and advanced atherosclerosis has been made. Given the strong association of OSAS with cardiovascular and cerebrovascular morbidity, measurement tools for endothelial dysfunction should be brought to the clinic to optimally treat patients with OSAS. Improved OSAS recognition and appropriate CPAP therapy may help to prevent vascular complications and improve the care of OSAS patients.

\section{References}

1. Verstraeten E (2007) Neurocognitive effects of obstructive sleep apnea syndrome. Curr Neurol Neurosci Rep 7: 161-166.

2. Marin JM, Carrizo SJ, Vicente E, Agusti AG (2005) Long-term cardiovascular outcomes in men with obstructive sleep apnoea-hypopnoea with or without treatment with continuous positive airway pressure: an observational study. Lancet 365: 1046-1053.

3. Shahar E, Whitney CW, Redline S, Lee ET, Newman AB, et al. (2001) Sleepdisordered breathing and cardiovascular disease: cross-sectional results of the Sleep Heart Health Study. Am J Respir Crit Care Med 163: 19-25.

4. Meslier N, Gagnadoux F, Giraud P, Person C, Ouksel H, et al. (2003) Impaired glucose-insulin metabolism in males with obstructive sleep apnoea syndrome. Eur Respir J 22: 156-160

5. Ip MS, Tse HF, Lam B, Tsang KW, Lam WK (2004) Endothelial function in obstructive sleep apnea and response to treatment. Am J Respir Crit Care Med 169: 348-353.

6. Nieto FJ, Herrington DM, Redline S, Benjamin EJ, Robbins JA (2004) Sleep apnea and markers of vascular endothelial function in a large community sample of older adults. Am J Respir Crit Care Med 169: 354-360.

7. Ten VS, Pinsky DJ (2002) Endothelial response to hypoxia: physiologic adaptation and pathologic dysfunction. Curr Opin Crit Care 8: 242-250.

8. Ryan S, Taylor CT, McNicholas WT (2005) Selective activation of inflammatory pathways by intermittent hypoxia in obstructive sleep apnea syndrome. Circulation 112: 2660-2667

9. Polotsky VY, Savransky V, Bevans-Fonti S, Reinke C, Li J, et al. (2010) Intermittent and Sustained Hypoxia Induce a Similar Gene Expression Profile in the Human Aortic Endothelial Cells. Physiol Genomics. 
Citation: Lal C, Strange C (2011) Obstructive Sleep Apnea Syndrome, Hypoxemia and Endothelial Dysfunction: One Disease or Many? J Pulmonar Respirat Med 1:101. doi:10.4172/2161-105X.1000101

10. Budhiraja R, Parthasarathy S, Quan SF (2007) Endothelial dysfunction in obstructive sleep apnea. J Clin Sleep Med 3: 409-415.

11. Jelic S, Lederer DJ, Adams T, Padeletti M, Colombo PC, et al. (2010) Vascular inflammation in obesity and sleep apnea. Circulation 121: 1014-1021.

12. Ohga E, Nagase T, Tomita T, Teramoto S, Matsuse T, et al. (1999) Increased levels of circulating ICAM-1, VCAM-1, and L-selectin in obstructive sleep apnea syndrome. J Applied Physiol 87: 10-14.

13. Akishita M, Ohike Y, Yamaguchi Y, lijima K, Eto M, et al. (2011) Obstructive sleep apnea exacerbates endothelial dysfunction in people with metabolic syndrome. J Am Geriatr Soc 59: 1565-1566.

14. Saito T, Saito T, Sugiyama S, Asai K, Yasutake M, et al. (2010) Effects of longterm treatment for obstructive sleep apnea on pulse wave velocity. Hypertens Res 33: 844-849.

15. Yun $\mathrm{CH}$, Jung KH, Chu K, Kim SH, Ji KH, et al. (2010) Increased circulating endothelial microparticles and carotid atherosclerosis in obstructive sleep apnea. J Clin Neurol 6: 89-98.

16. Kheirandish-Gozal L, Bhattacharjee R, Kim J, Clair HB, Gozal D (2010) Endothelial progenitor cells and vascular dysfunction in children with obstructive sleep apnea. Am J Respir Crit Care Med 182: 92-97.

17. Berger S, Lavie L (2011) Endothelial progenitor cells in cardiovascular disease and hypoxia--potential implications to obstructive sleep apnea. Transl Res 158: $1-13$.

18. Partinen M, Palomaki H (1985) Snoring and cerebral infarction. Lancet 2: 1325-

19. Palomaki H, Partinen M, Juvela S, Kaste M (1989) Snoring as a risk factor for sleep-related brain infarction. Stroke 20: 1311-1315.

20. Spriggs DA, French JM, Murdy JM, Curless RH, Bates D, et al. (1992) Snoring increases the risk of stroke and adversely affects prognosis. Q J Med 83: 555562.
21. Neau JP, Meurice JC, Paquereau J, Chavagnat JJ, Ingrand P, et al. (1995) Habitual snoring as a risk factor for brain infarction. Acta Neurol Scand 92 $63-68$

22. Hung J, Whitford EG, Parsons RW, Hillman DR (1990) Association of sleep apnoea with myocardial infarction in men. Lancet 336: 261-264.

23. Duchna HW, Stoohs R, Guilleminault C, Christine Anspach M, SchultzeWerninghaus $\mathrm{G}$ et al. (2006) Vascular endothelial dysfunction in patients with mild obstructive sleep apnea syndrome. Wien Med Wochenschr 156: 596-604

24. Chung S, Yoon IY, Lee CH, Kim JW (2010) The association of nocturnal hypoxemia with arterial stiffness and endothelial dysfunction in male patients with obstructive sleep apnea syndrome. Respiration 79: 363-369.

25. Kadohira T, Kobayashi Y, Iwata Y, Kitahara H, Komuro I (2011) Coronary artery endothelial dysfunction associated with sleep apnea. Angiology 62: 397-400.

26. Buchner NJ, Quack I, Woznowski M, Stähle C, Wenzel U, et al. (2011) Microvascular Endothelial Dysfunction in Obstructive Sleep Apnea Is Caused by Oxidative Stress and Improved by Continuous Positive Airway Pressure Therapy. Respiration 82: 409-417.

27. Ciccone MM, Favale S, Scicchitano P, Mangini F, Mitacchione G, et al. (2011) Reversibility of the endothelial dysfunction after CPAP therapy in OSAS patients. Int J Cardiol.

28. Parra O, Sánchez-Armengol A, Bonnin M, Arboix A, Campos-Rodríguez $\mathrm{F}$ et al. (2011) Early treatment of obstructive apnoea and stroke outcome: a randomised controlled trial. Eur Respir J 37: 1128-1136.

29. Gozal D, Kheirandish-Gozal L, Serpero LD, Sans Capdevila O, Dayyat E (2007) Obstructive sleep apnea and endothelial function in school-aged nonobese children: effect of adenotonsillectomy. Circulation 116: 2307-2314.

30. Bhattacharjee R, Alotaibi WH, Kheirandish-Gozal L, Capdevila OS, Gozal D (2010) Endothelial dysfunction in obese non-hypertensive children withou evidence of sleep disordered breathing. BMC pediatr 10: 8 . 